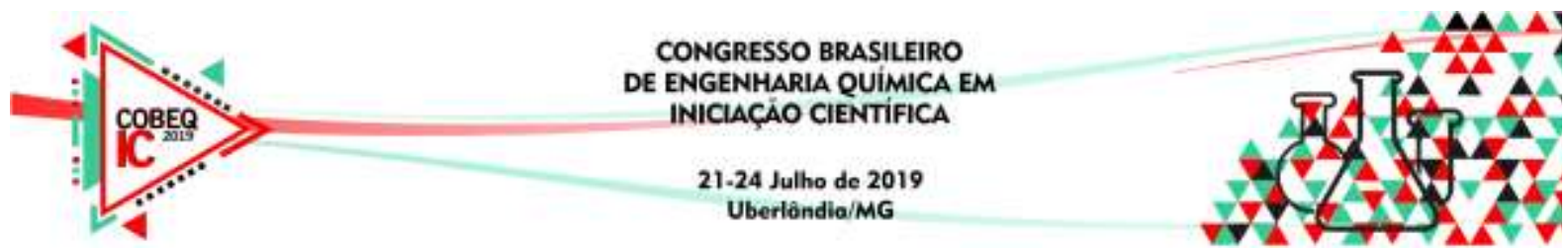

\title{
SÍNTESE DA ZEÓLITA ZSM-5 COM MISTURAS BINÁRIAS DE HIDRÓXIDO DE TETRAETILAMÔNIO E AMINAS COMO DIRECIONADORES ORGÂNICOS
}

\author{
D. P. S. SILVA, L. R. A. SARMENTO, B. J. B. SILVA, A. O. S. SILVA
}

Universidade Federal de Alagoas, Centro de Tecnologia, Departamento de Engenharia
Química, Laboratório de Síntese de Catalisadores - LSCat
E-mail para contato: diogo.silva@ctec.ufal.br

RESUMO - Dentre os materiais microporosos utilizados na catálise heterogênea, as zeólitas tornaram-se promissoras devido às pertinentes propriedades físicoquímica e catalítica. Destacando-se a zeólita ZSM-5 utilizada como catalisador em reações nas áreas petroquímica e química fina, como as reações de craqueamento, isomerização, alquilação e aromatização de olefinas. Diante do exposto, o objetivo deste trabalho foi sintetizar a zeólita ZSM-5, através de misturas binárias de hidróxido de tetraetilamônio (TEAOH) e aminas (n-butilamina, isobutilamina e diisobutilamina) atuando como agentes direcionadores de estrutura (SDA's). A difratometria de raios $\mathrm{X}$ indicou que apenas o sistema reacional contendo TEAOH e n-butilamina foi possível à obtenção da ZSM-5 altamente cristalina e isenta de fases concorrentes. As isotermas de adsorção-dessorção de nitrogênio indicaram a obtenção de um material com elevada área superficial e volume de poros. Dessa forma, foi possível identificar uma nova rota de síntese na obtenção da zeólita ZSM-5.

\section{INTRODUÇÃO}

As zeólitas são polímeros inorgânicos complexos e cristalinos, baseadas num arranjo tridimensional, constituído por tetraedros $\mathrm{TO}_{4}(\mathrm{~T}=\mathrm{Si}$ ou $\mathrm{Al})$ interconectados através de um átomo de oxigênio em comum (Englert e Rubio, 2005). Devido a propriedades intrínsecas das zeólitas, como cristalinidade, grande área superficial, acidez, capacidade de troca iônica e seletividade, estas são utilizadas em uma variedade de aplicações como: processos de separação de gases, catálise, adsorventes e trocadores iônicos de alta capacidade e seletividade (Chen et al., 2018).

A ZSM-5 é uma zeólita que se caracteriza pelo alto teor de silício na estrutura (razão $\mathrm{Si} / \mathrm{Al}$ entre 11 e infinito), pertence ao grupo da família pentasil e possui estrutura tipo MFI de acordo com a classificação proposta pela International Zeolite Association (IZA) (Chen et al., 2018). É utilizada como catalisador na indústria petroquímica em processos de isomerização, alquilação e aromatização (Cundy e Fox, 2005).

As moléculas dos direcionadores orgânicos afetam significativamente a cristalização das zeólitas por: (i) influenciar o processo de gelificação e/ou nucleação, onde as unidades $\mathrm{TO}_{4}$ são organizadas numa geometria particular em torno de si mesmas e, como resultado, 


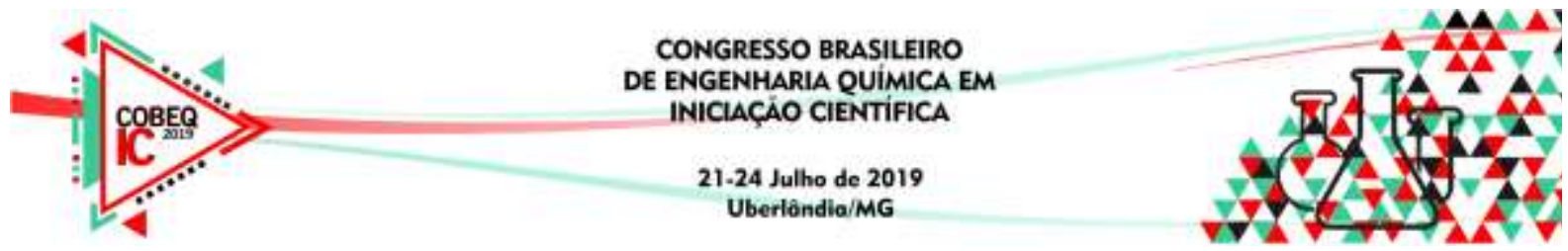

proporcionam espécies precursoras para subsequente nucleação ou crescimento dos cristais (Zhang et al., 2012); e (ii) diminuir o potencial químico da rede formada através da inclusão do direcionador durante a síntese (Yu, 2007).

Com base no que foi exposto, fica evidente que a adição de direcionadores orgânicos ao meio reacional empregado para a síntese de zeólitas desempenha um papel fundamental na determinação da estrutura que será formada durante o processo de cristalização. Dessa forma, o presente trabalho avaliou o efeito das propriedades texturais das zeólitas ZSM-5 sintetizadas em sistemas reacionais contendo misturas binárias de direcionadores orgânicos. Foi analisado o papel da adição do hidróxido de tetraetilamônio e de aminas (n-butilamina, isobutilamina e diisobutilamina) sobre o tipo de fase cristalina obtida, a velocidade de cristalização e o efeito da composição da mistura reacional sobre o processo de cristalização dos materiais zeolíticos. Todas as amostras foram caracterizadas por difratometria de raios $\mathrm{X}(\mathrm{DRX})$ e as amostras mais cristalinas isentas de contaminantes foram caracterizadas adicionalmente por análises térmicas (TG/DTG) e adsorção-dessorção de nitrogênio.

\section{METODOLOGIA}

\subsection{Síntese das zeólitas}

As composições e o procedimento de preparo dos géis de síntese foram baseados no método hidrotermal clássico (Szostak, 1998). Os reagentes precursores foram misturados a partir da seguinte composição molar: 0,2 TEAOH : 0,8 AMINA : $1 \mathrm{SiO}_{2}: 0,01 \mathrm{Al}_{2} \mathrm{O}_{3}: 0,3$ $\mathrm{Na}_{2} \mathrm{O}: 20 \mathrm{H}_{2} \mathrm{O}: 0,3 \mathrm{H}_{2} \mathrm{SO}_{4}$, as aminas utilizadas foram: n-butilamina, isobutilamina e diisobutilamina.

O procedimento de mistura dos materiais precursores foi realizado em etapas: (i) dissolução do silicato de sódio em $40 \%$ da água, posterior, adição do sulfato de alumínio octadecahidratado, sob de agitação por 20 minutos (solução A), (ii) diluição do ácido sulfúrico em $60 \%$ da água e a adição dos direcionadores orgânicos (TEAOH e amina), sob agitação por 20 minutos (solução B), (iii) mistura das soluções A e B, sob agitação por 30 minutos. Após o preparo do gel, este foi transferido para vasos de teflon revestidos em autoclaves de aço inoxidável, e aquecidos em estufas a $170{ }^{\circ} \mathrm{C}$ durante 24 a 96 horas. $\mathrm{O}$ sólido resultante foi separado do líquido sobrenadante por filtração a vácuo, sendo lavado com água destilada até atingir $\mathrm{pH}$ neutro, por fim, seco em estufa a $120^{\circ} \mathrm{C}$ por 12 horas. Os catalisadores foram calcinados sob fluxo de ar de $100 \mathrm{~mL} \cdot \mathrm{min}^{-1}$ com uma rampa de aquecimento de $2{ }^{\circ} \mathrm{C} \cdot \mathrm{min}^{-1}$ até $550{ }^{\circ} \mathrm{C}$ por 6 horas.

\subsection{Caracterizações}

As análises de DRX foram realizadas utilizando um difratômetro da Shimadzu XRD6000 , com radiação $\mathrm{CuK} \alpha(\lambda=0,1542 \mathrm{~nm})$, filtro de $\mathrm{Ni}$, voltagem de $40 \mathrm{kV}$ e corrente de $30 \mathrm{~mA}$. A aquisição dos dados foi realizada no intervalo de $2 \theta$ entre 3 e $40^{\circ}$, com velocidade de varredura de $2^{\circ} \cdot \min ^{-1}$ e passo de $0,02^{\circ}$. As análises térmicas (TG/DTG) foram realizadas em uma termobalança da Shimadzu DTG-60H, com taxa de aquecimento $10^{\circ} \mathrm{C} \cdot \mathrm{min}^{-1}$, sob atmosfera de ar sintético de $100 \mathrm{~mL} \cdot \mathrm{min}^{-1}$, de temperatura ambiente à $900{ }^{\circ} \mathrm{C}$. As medidas de 


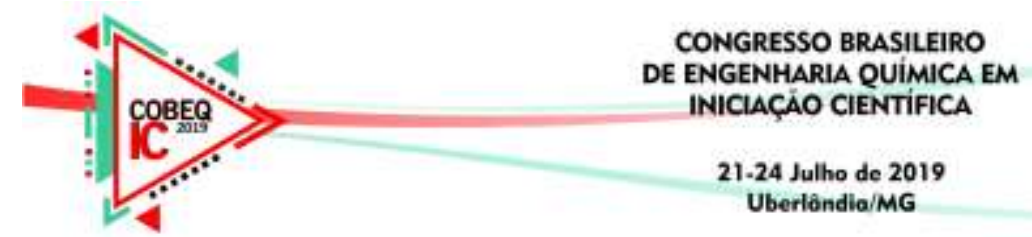

adsorção-dessorção de nitrogênio foram realizadas em um equipamento Micromeritics ASAP 2020, no intervalo de pressões relativas $\mathrm{P} / \mathrm{P}_{0}$ entre 0,01 e 1,0 .

\section{RESULTADOS E DISCUSSÕES}

A Figura 1 ilustra os perfis de DRX das amostras sintetizadas utilizando misturas de TEAOH e das aminas em diferentes tempos.

Figura 1 - Difratogramas comparativos utilizando misturas do TEAOH e (a) diisobutilamina, (b) isobutilamina, (c) n-butilamina como direcionadores orgânicos, em diferentes tempos.
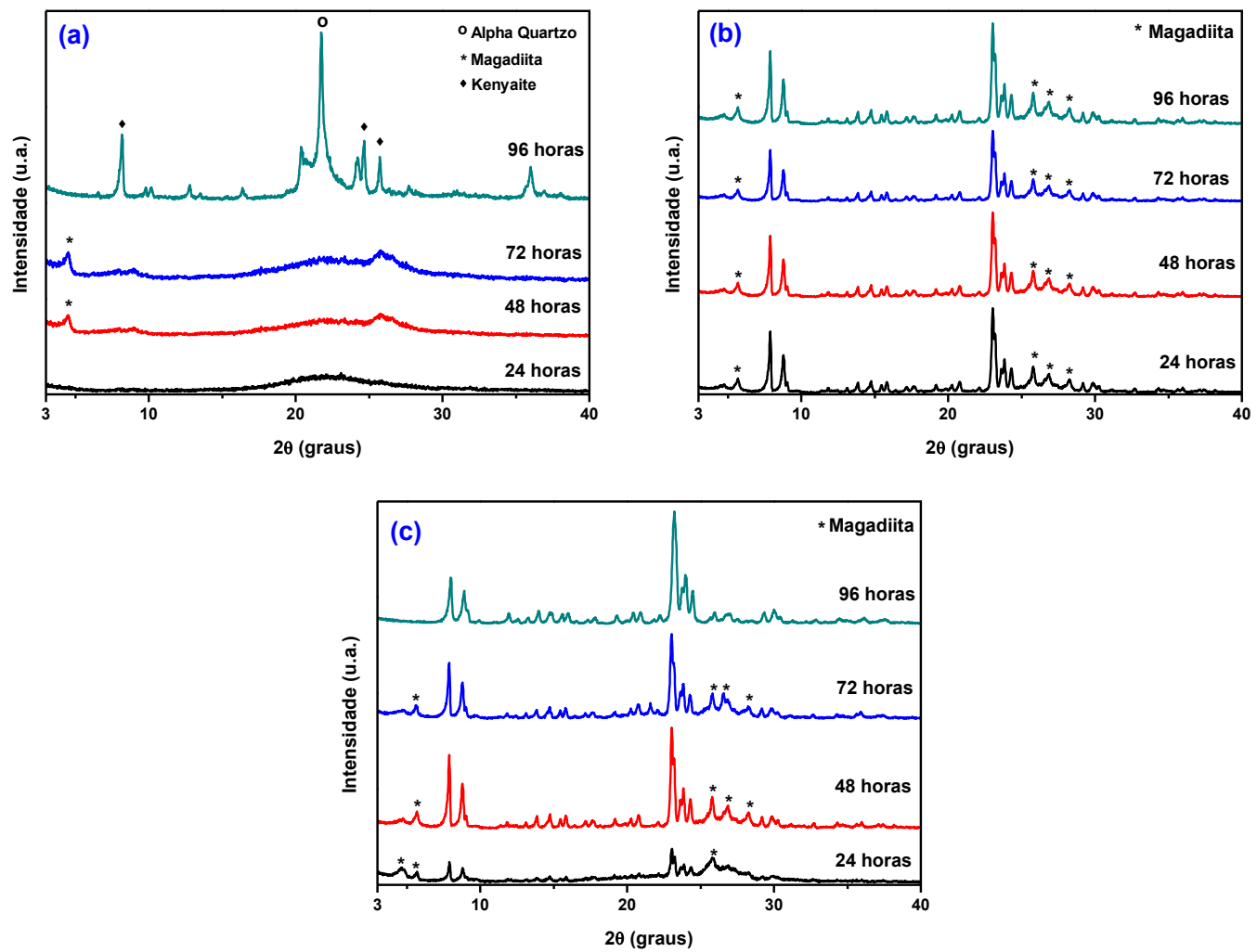

A Figura 1 (a) mostra os difratogramas das amostras sintetizadas com misturas binárias de TEOH e isobutilamina, onde não se verificou a formação de fases cristalinas com 24 horas, havendo o surgimento de picos de difração a partir de 48 e 72 horas atribuídos a fase lamelar Kenyaita (JCPDS N²0-1157), por fim, com 96 horas há a formação fase lamelar Magadiita (JCPDS $\mathrm{N}^{\circ} 42-1350$ ) concomitantemente com a fase condensada alpha quartzo (JCPDS $\mathrm{N}^{\circ}$ 46-1045).

A Figura 1(b) apresenta os difratogramas das amostras sintetizadas com misturas binárias de TEAOH e isobutilamina, os quais demonstraram que em todos os períodos de cristalização são obtidos materiais com a coexistência da fase da ZSM-5 (JCPDS No 42-0023) e a da fase lamelar Magadiita. Estudos relatam a síntese da Magadiita, com a proporção $\mathrm{SiO}_{2}: \mathrm{NaOH}$ na faixa de $1,0: 0,2$ a $1,0: 9,0$, onde a proporção $\mathrm{SiO}_{2}: \mathrm{NaOH}$ utilizada neste trabalho foi de $1,0: 0,3$, podendo então a quantidade de $\mathrm{NaOH}$ utilizada ter favorecido a 


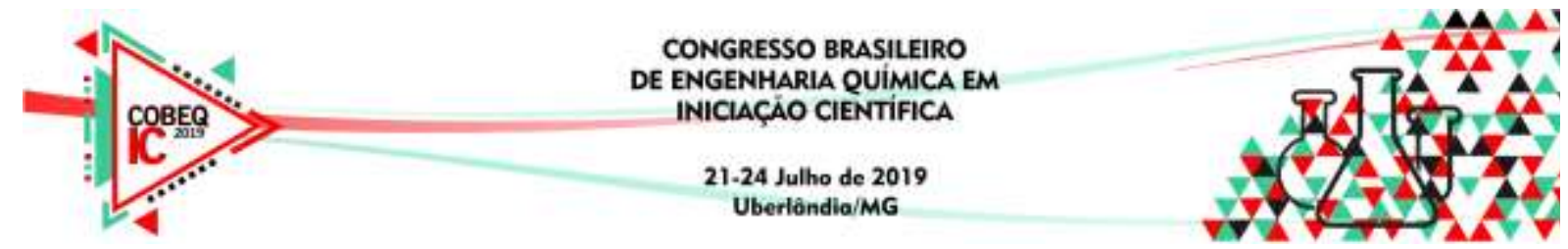

formação da magadiita, levando à formação de várias áreas segregadas classificadas em regiões zeolíticas e lamelares (Ozawa et al., 2010, Xu et al., 2015).

Os espectros da Figura 1 (c) demonstram que até 72 horas de cristalização são obtidos materiais com a coexistência da ZSM-5 e a fase lamelar Magadiita, sendo obtida a fase da ZSM-5 isenta de fases concorrentes a partir de 96 horas. A partir das reflexões de Bragg, constatou-se que o material obtido apresentou um difratograma típico dessa zeólita em comparação com o padrão simulado de DRX da estrutura MFI-IZA (Razavian e Fatemi, 2015). Isso indica que os métodos de sínteses empregados foram efetivos para a obtenção da zeólita ZSM-5 com a mistura binária de TEAOH e n-butilamina.

A Figura 2 exibe as curvas de TG/DTG da amostra de ZSM-5 sintetizada com mistura binária de TEAOH e n-butilamina com 96 horas de cristalização. Através das curvas de DTG identificaram-se quatro eventos de perdas de massas distintos. Podendo-se atribuí-los a: (I) e (II) de $25{ }^{\circ} \mathrm{C}-360{ }^{\circ} \mathrm{C}$, compreende a perda de massa relacionada à eliminação da água fisissorvida (evaporação de grupos hidroxilas ligadas fracamente às partículas); (III) a região entre $360{ }^{\circ} \mathrm{C}-530{ }^{\circ} \mathrm{C}$, corresponde a decomposição oxidativa de íons amônio provenientes do agente direcionador de estrutura (SILVA et al., 2008); (IV) acima de $530{ }^{\circ} \mathrm{C}$ perda de massa é decorrente do coque depositado sobre o catalisador gerado durante a queima do direcionador.

Figura 2 - Análise térmica da zeólita ZSM-5 sintetizada com mistura binária de TEAOH e n-butilamina, com 96 horas de cristalização.

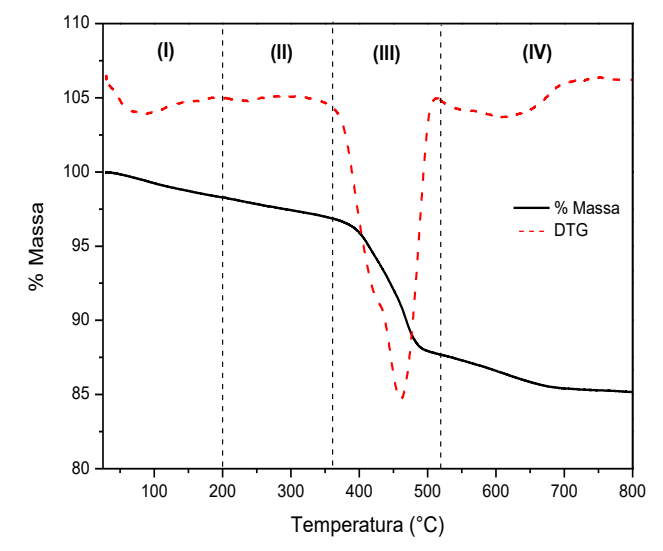

A Figura 3 apresenta a isoterma de adsorção-dessorção de nitrogênio sobre a amostra de ZSM-5 sintetizada com mistura binária de TEAOH e n-butilamina, como direcionadores estruturais. De acordo com a classificação da International Union of Pure and Applied Chemistry (IUPAC), essa medida mostra que o material obtido adsorve o nitrogênio com uma combinação de isoterma do tipo I, característico de adsorventes microporoso, com a do tipo IV, característicos de sólidos mesoporosos com a presença de um loop de histerese, que está relacionado com a formação de poros em formas de fenda (Senapati, 2014).

A Tabela 1 apresenta os dados das propriedades texturais da zeólita ZSM-5 sintetizada com misturas binárias de direcionadores estruturais. $\mathrm{O}$ valor da área específica calculada pelo método BET foi elevada, característico de sólidos microcoporos, o que está de acordo com valores relatados na literatura para zeólitas do tipo ZSM-5 (Zhang et al., 2012; Yu, 2007). A 


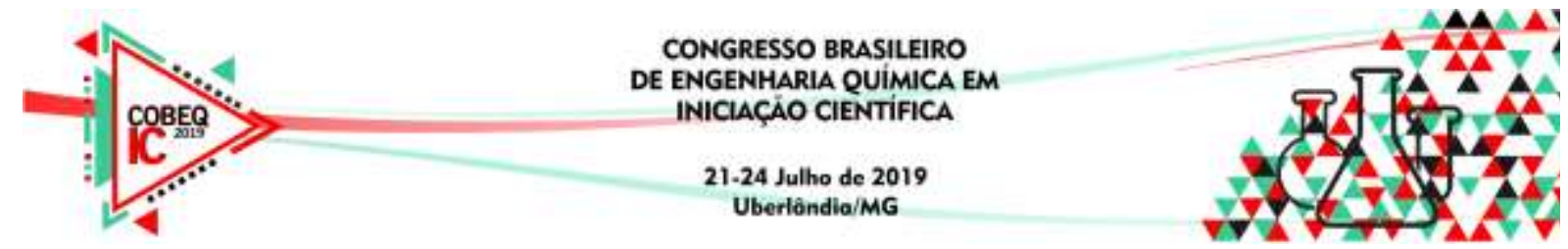

presença de mesoporos caracterizados pelo loop de histerese na isoterma de adsorção foi comprovada pelo pertinente volume de mesoporos, o que concorda com a classificação da histerese observada na Figura 3.

Tabela 1 - Propriedades texturais da ZSM-5 sintetizada com mistura binária de TEAOH e n-butilamina com 96 horas de cristalização.

\begin{tabular}{|c|c|c|c|c|}
\hline $\begin{array}{c}A_{\mathrm{BET}} \\
\left(\mathbf{m}^{2} \cdot \mathbf{g}^{-1}\right)\end{array}$ & $\begin{array}{c}\mathbf{A}_{\mathrm{EX}} \\
\left(\mathbf{m}^{2} \cdot \mathbf{g}^{-1}\right)\end{array}$ & $\begin{array}{c}\mathbf{A}_{\text {Micro }} \\
\left(\mathbf{m}^{2} \cdot \mathbf{g}^{-1}\right)\end{array}$ & $\begin{array}{c}\mathbf{V}_{\text {Total }} \\
\left(\mathbf{c m}^{3} \cdot \mathbf{g}^{-1}\right)\end{array}$ & $\begin{array}{c}\mathbf{V}_{\text {Micro }} \\
\left(\mathbf{c m}^{3} \cdot \mathbf{g}^{-1}\right)\end{array}$ \\
\hline 329 & 109 & 233 & 0,26 & 0,12 \\
\hline
\end{tabular}

Figura 3 - Isoterma de adsorção-dessorção de $\mathrm{N}_{2}$ da zeólita ZSM-5 sintetizada com mistura binária de TEAOH e n-btutilamina, com 96 horas de cristalização.

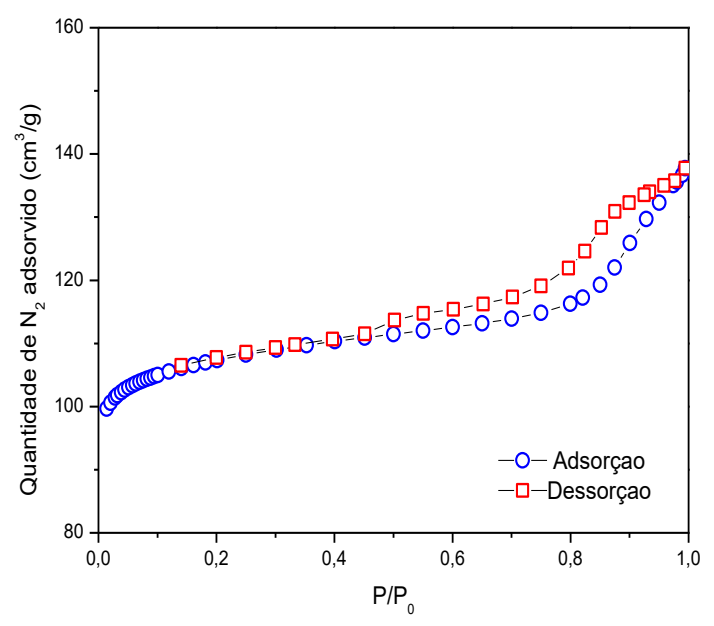

\section{CONCLUSÃO}

As análises de DRX indicaram que apenas o sistema reacional contendo mistura binária do TEAOH e da n-butilamina, como SDA's, com 96 horas de cristalização, foi possível obter a ZSM-5, sem que fosse constatada a presença de fases contaminantes. As isotermas de adsorção-dessorção de nitrogênio indicaram a obtenção de um material com elevado valor de área superficial e volume de mesoporos. Dessa forma, foi possível identificar uma nova rota de síntese na obtenção da ZSM-5.

\section{REFERÊNCIAS BIBLIOGRÁFICAS}

CHEN, Y.; LI, C.; CHEN, X.; LIU, Y.; LIANG, C. Synthesis of ZSM-23 zeolite with duas structure direting agents for hydroisomerization of n-hexadecane. Micro. Meso. Mat., v. 80, p. 216-224, 2018.

ENGLERT, A. H.; RUBIO, J. Characterization and environmental application of a chilean natural zeólita. Int. Jour. Min. Proc., v. 75, p. 2129, 2005. 


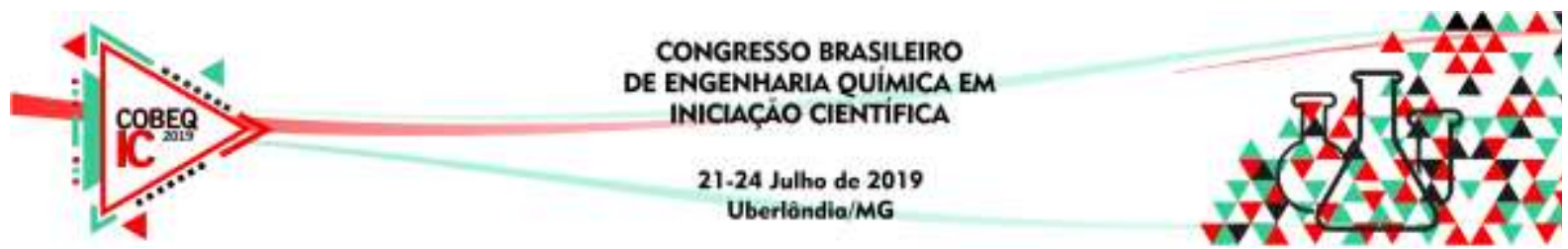

OZAWA, K.; OKADA, $\quad$ R.; NAKAO, Y.; OGIWARA, T.; ITOH, H.; ISO, F. Hydrothermal Synthesis of Magadiite/Si-ZSM-11 Composites, Jour. Americ. Cera. Soc., v. 93, p. 4022-4025, 2010.

RAZAVIAN, M.; FATEMI, S. Synthesis and application of ZSM-5/SAPO-34 and SAPO34/ZSM-5 composite systems for propylene yield enhancement in propane dehydrogenation process. Micro. Meso. Mat., v. 201, p. 176 - 189, 2015.

SENAPATI, S. Post-synthetic modifications of as-made zeolite frameworks near the structuredirecting agents. Jour. Mat. Chem., p. 10470 - 10484, 2014.

SILVA, J. B.; RODRIGUES, J. A. J.; NONO, M. DO C. A. Caracterização de materiais catalíticos. 2008. 69 f. Pós-Graduação em Engenharia e Tecnologia Espaciais, Instituto Nacional de Pesquisas Espaciais, São José dos Campos - SP, 2008.

CUNDY, C. S.; COX, P. A. The hydrothermal synthesis of zeolites: precursors, intermediates and reaction mechanism. Micro. Meso. Mat., v. 82, p. 1-78. 2005.

XU, B.; ZHU, X.; CAO, Z.; YANG, L.; YANG, W. Catalytic oxidative dehydrogenation of nbutane over $\mathrm{V}_{2} \mathrm{O}_{5} / \mathrm{MO}-\mathrm{Al}_{2} \mathrm{O}_{3}(\mathrm{M}=\mathrm{Mg}, \mathrm{Ca}, \mathrm{Sr}, \mathrm{Ba})$ catalysts. Chin. Jour. Cat., v. 36, p. 1060-1067, 2015.

YU, J. Synthesis of zeolite. In: ĈEJKA, J.; VAN BEKKUM, H.; CORMA, A.; SCHUTH, F. (eds). Introduction to zeolite science and practice. 3a ed. Rev. Amst., p. 39-103. 2007.

ZHANG, L.; YANG, W.; LIU, Y.; LIANG, C. Organic template-free synthesis of ZSM5/ZSM-11 co-crystalline zeolite. Micro. Meso. Mat., v. 147, p. 117-126, 2012.

ZSOSTAK, R., Molecular Sieves: Principles of Synthesis and Identification, $2^{\circ}$ Ed., Blackie Academic \& Professional, London, 1998. 